\title{
Effect of graphene oxide nanomaterial in electroclinic liquid crystals
}

\author{
A. Malik, ${ }^{1,2}$ A. Choudhary, ${ }^{1}$ P. Silotia, ${ }^{2}$ A. M. Biradar, ${ }^{1, a}$ V. K. Singh, ${ }^{3}$ and N. Kumar ${ }^{3}$ \\ ${ }^{1}$ Liquid Crystal and Self Assembled Monolayer Section, National Physical Laboratory, \\ Dr. K. S. Krishnan Road, New Delhi 110012, India \\ ${ }^{2}$ Department of Physics and Astrophysics, University of Delhi, Delhi 110007, India \\ ${ }^{3}$ Defence Laboratory, Jodhpur, Rajasthan 342011, India
}

(Received 11 August 2010; accepted 3 November 2010; published online 29 December 2010)

\begin{abstract}
The collective dielectric relaxation studies have been carried out on the electroclinic liquid crystals (ELCs) doped with 0.1 to $0.2 \mathrm{wt} \%$ of graphene oxide (GO) in the frequency range of $20 \mathrm{~Hz}$ to 1 $\mathrm{MHz}$. The GO favors for a good quality vertical alignment without any surface treatment of the substrates. The coupling of GO with indium tin oxide (ITO) substrate and ELC materials affects the molecular ordering and supports the ELC molecules to be aligned along the GO attached to the ITO surface in vertical direction. The vertical alignment can be changed to homogeneous by applying a high bias field to the sample and such converted homogeneous cell shows an additional dielectric relaxation peak in the low frequency side of Goldstone mode in $\mathrm{SmC}^{*}$ phase due to presence of $\mathrm{GO}$ whereas in the pure material no such peak was observed. The frequency separation of both peaks (Goldstone mode and an additional peak) increases with temperature and low frequency peak vanishes near transition temperature. (C) 2010 American Institute of Physics.
\end{abstract}

[doi:10.1063/1.3524540]

\section{INTRODUCTION}

Much of the research in the field of liquid crystals (LCs) focuses around the ferroelectric LCs (FLCs) since their discovery by Meyer et al. ${ }^{1}$ Theoretical as well as experimental work have been devoted to the study of FLCs in Smectic $\mathrm{C}^{*}\left(\mathrm{SmC}^{*}\right)$ to Smectic $\mathrm{A}^{*}\left(\mathrm{SmA}^{*}\right)$ phase transitions. ${ }^{2-5}$ Among all FLCs, a special class of FLC with considerably wide range of $\mathrm{SmA}^{*}$ phase and a large electroclinic coefficient, is very interesting ${ }^{6,7}$ due to its ultra fast response and nonlayer shrinkage. ${ }^{8-12}$ These FLCs are known as electroclinic LCs (ELCs).

To investigate the dielectric relaxation behaviors of ELCs in $\mathrm{SmC}^{*}$ phase, one can give details of two low frequency modes, the Goldstone and soft modes which are related to the structural changes at the $\mathrm{SmA}^{*}-\mathrm{SmC}^{*}$ phase transition. Goldstone mode arises due to the phase fluctuations of azimuthal angle and the soft mode is due to the fluctuation in the amplitude of tilt angle. ${ }^{2,13}$ These two modes describe completely the low frequency dynamics of the $\mathrm{SmC}^{*}$ phase in the ideal infinite samples.

Moreover, there may be some additional dynamic modes produced by the dynamics of the molecules or by the molecular interaction between the molecules of the ELCs and some dopant materials. ${ }^{14,15}$ The doping of various nanomaterials in to LCs has enhanced many features of LCs in the form of frequency modulation response, nonvolatile memory effect, faster electro-optical response, low driving voltage and enhanced photoluminescence. ${ }^{16-20}$ Now a days, graphene and graphene oxide (GO) are the interesting nanomaterial for dynamic research studies. GO can be prepared by extensive chemical attacks of graphite crystal to introduce oxygen. $^{21}$ The ease of synthesizing GO and its solution pro-

${ }^{\text {a)} E l e c t r o n i c ~ m a i l: ~ a b i r a d a r @ m a i l . n p l i n d i a . e r n e t . i n . ~}$ cessing compatibility make the material attractive for large area applications including transparent conductors, ${ }^{22-25}$ electrical energy storage devices and polymer composites. ${ }^{26}$ In 2008, Blake et $a .^{27}$ have used graphene to fabricate electrodes for the LC device and showed excellent performance with a high contrast ratio. Recently, in the beginning of 2010, Safavi and Tohidi ${ }^{28}$ designed and characterized the LCgraphite composite electrodes and found to be perfect for the application of electric field in different electrochemical and biosensing applications. GO is an intriguing nanomaterial and shows very high anisotropic morphology. Becerril et al. evaluated the potential of highly reduced GO thin films as transparent conductors ${ }^{22}$ and proposed a possible route for addressing this issue. GO is a promising precursor for preparing graphene based composites and electronic applications. $^{29}$

Up till now, graphene and GO have mainly been used in the fabrication of electrodes and applied in the form of thin film. However doping of GO in LCs is rarely reported in literature. In the present study, we have doped the GO nanomaterial in ELC material. With the objective of studying the collective dielectric processes in the GO doped ELC material, the experiments have been carried out on vertical and planar converted samples using the same sample cell. It has been observed that by the addition of GO, a low frequency peak along with the Goldstone mode in the $\mathrm{SmC}^{*}$ phase has been observed near the transition temperature $\left(\mathrm{T}_{\mathrm{c}}\right)$ of ferroto paraelectric phase in a converted planar cell. This low frequency mode is strongly temperature and electric field dependent.

\section{EXPERIMENT}

GO used in the present study was synthesized by Hummer's method, ${ }^{21}$ only the purification step has been replaced 
with washing of products step with known concentration of $\mathrm{HNO}_{3}$. One can remove the impurities of other metal ions and enhance the oxidation of graphite by this replacement. The product was thoroughly washed with distilled water and a golden yellow powder of GO nanomaterial was obtained. The structural characterization of GO was carried out in detail $^{30}$ by powder $\mathrm{x}$-ray diffraction, Fourier transform infrared, and atomic force microscopy (AFM). AFM images of GO nanomaterial showed layering morphology having thickness of $\sim 25 \mathrm{~nm}$ consisting of each stack thickness of $\sim 5 \mathrm{~nm}$. For the dielectric relaxation processes and electrooptical studies of the ELC material, highly conducting indium tin oxide (ITO) with a sheet resistance of 18-20 $\Omega / \square$ coated optically flat glass plates were used as electrodes. The electrodes in the form of square pattern $\left(4.5 \times 4.5 \mathrm{~mm}^{2}\right)$ were designed using a negative photolithography technique. The glass substrates were assembled without any surface treatment, facing the electrodes to each other, in the form of cell maintaining a uniform thickness of around $6 \mu \mathrm{m}$ by using Mylar spacers. ${ }^{31}$

In the present study, we have used a commercially available ELC BDH $764 \mathrm{E}$ having phase sequence as follows:

$$
\begin{gathered}
-7^{\circ} \mathrm{C} \quad 28^{\circ} \mathrm{C} \text { SmC }^{*} \leftrightarrow 3^{\circ} \mathrm{C} \quad 89-92{ }^{\circ} \mathrm{C} \\
\text { Cryst } \leftrightarrow \mathrm{Sm}^{*} \leftrightarrow \mathrm{N} \leftrightarrow \text { Iso. }
\end{gathered}
$$

A small amount (0.1-0.2 wt \%) of GO was dispersed in the ELC material at room temperature and then the composition was introduced into the LC sample cells in isotropic phase of the ELC material by means of capillary action.

The molecular and collective dynamic dielectric processes were investigated by broadband dielectric spectroscopy in an electrically shielded parallel plate condenser using impedance analyzer 6540A (Wayne Kerr, U.K.) in the frequency range of $20 \mathrm{~Hz}$ to $1 \mathrm{MHz}$ and the probe amplitude for the dielectric measurement was fixed $0.5 \mathrm{~V}$. The optical micrograph of the ELC material was recorded using a polarizing optical microscope (AX-40, Carl Zeiss, Germany) fitted with a computer controlled camera. The material constants such as polarization $\left(\mathrm{P}_{\mathrm{s}}\right)$ and rotational viscosity $(\eta)$ have been determined by using an automatic LC tester (ALCT, Instec, U.S.A.) which works on the principle of polarization reversal current peak due to triangular wave method. The temperature controller (JULABO F-25 HE) equipment with temperature stability of $\pm 0.01{ }^{\circ} \mathrm{C}$ was used for controlling the temperature of the sample cell. The sample holder was kept isolated from the external sources (electrical and thermal).

\section{RESULTS AND DISCUSSIONS}

Dielectric spectroscopy is very effective tool to detect stochastic reorientation of collective molecular dipole moment processes particularly in $1 \mathrm{~Hz}$ to $1 \mathrm{MHz}$ frequency domain of dielectric spectrum. ${ }^{32}$ Fig. 1 reveals the real part of dielectric permittivity $\left(\varepsilon^{\prime}\right)$ in vertical (in virgin sample) and planar aligned (aligned by dc electric field) sample with their corresponding optical textures as an inset of this figure. Vertical alignment is the result of filling the GO added ELC in a sample cell having untreated ITO coated glass substrates. The molecular alignment has been found vertical in

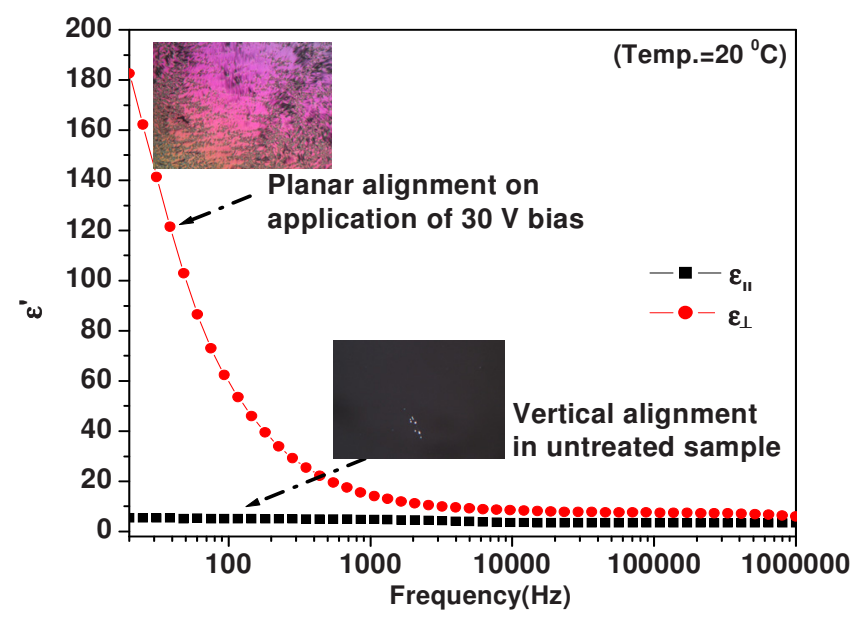

FIG. 1. (Color online) Dielectric permittivity $\left(\varepsilon^{\prime}\right)$ as a function of frequency in vertical (virgin sample) and planar aligned (dc electric field treated) sample cell at $20{ }^{\circ} \mathrm{C}$ temperature with their corresponding optical micrographs as an inset for GO doped ELC (BDH 764E) sample cell.

virgin sample cell; therefore, the dielectric permittivity value $\varepsilon^{\prime}$ in low frequency domain is lower as compared to the planar aligned sample. The decrease in $\varepsilon^{\prime}$ value is due to the low dielectric strength along the long molecular axis. The vertical alignment was also observed by Kumar et al. ${ }^{33}$ by doping polymeric nanoparticles in ELCs. The planar alignment was achieved by applying dc electric field of $30 \mathrm{~V}$, as can be seen in Fig. 1. It is worth to be noted here that once the sample goes in the planar configuration then it cannot go back to the vertical alignment which means that the planar to vertical transition is not reversible. The brightness of the optical texture confirms the conversion of planar alignment. Its corresponding $\varepsilon^{\prime}$ curve of planar aligned sample as a function of frequency shows the higher dielectric value $\varepsilon^{\prime}$ as compared to vertical aligned sample. In general, such complex dielectric permittivity behaviors can be studied by the relation

$$
\varepsilon^{*}(\omega)=\varepsilon_{\infty}+\sum_{i=\mathrm{G}, \mathrm{S}} \frac{\left(\varepsilon_{o}^{i}-\varepsilon_{\infty}^{i}\right)}{1-\left(i \omega \tau_{i}\right)^{1+\alpha_{i}}},
$$

where $\alpha$ stands for the distribution parameter. $\mathrm{G}$ and $\mathrm{S}$ stands for Goldstone and soft mode, respectively. The real and imaginary part can be separated out easily from the complex function for convenience.

The dielectric investigation of planar converted alignment of GO doped ELC system has been carried out where the real part of complex permittivity, $\varepsilon^{\prime}$ is plotted as a function of temperature at selective frequencies, as shown in Fig. 2. Its comparative study with pure ELC at same parameters is shown in inset of Fig. 2. The curve of $\varepsilon^{\prime}$ of GO doped ELC at $20 \mathrm{~Hz}$ frequency shows the faster decreasing trend near $\mathrm{T}_{\mathrm{c}}$ of $\mathrm{SmC}^{*}$ to $\mathrm{SmA}^{*}$ phase, in comparison to pure ELC. The $\varepsilon^{\prime}$ drops to very low value (below 25) in $\mathrm{SmA}^{*}$ phase in GO doped ELC, while it remains high $(\sim 50)$ in case of pure ELC sample as shown in inset of Fig. 2. Another noticeable behavior has been observed near transition temperature $\left(26-27^{\circ} \mathrm{C}\right)$ of GO doped ELC at $1 \mathrm{kHz}$ frequency is that the $\varepsilon^{\prime}$ value at this frequency is still remains high near $T_{c}$ and drops to very low in $\mathrm{SmA}^{*}$ phase, whereas it is almost con- 


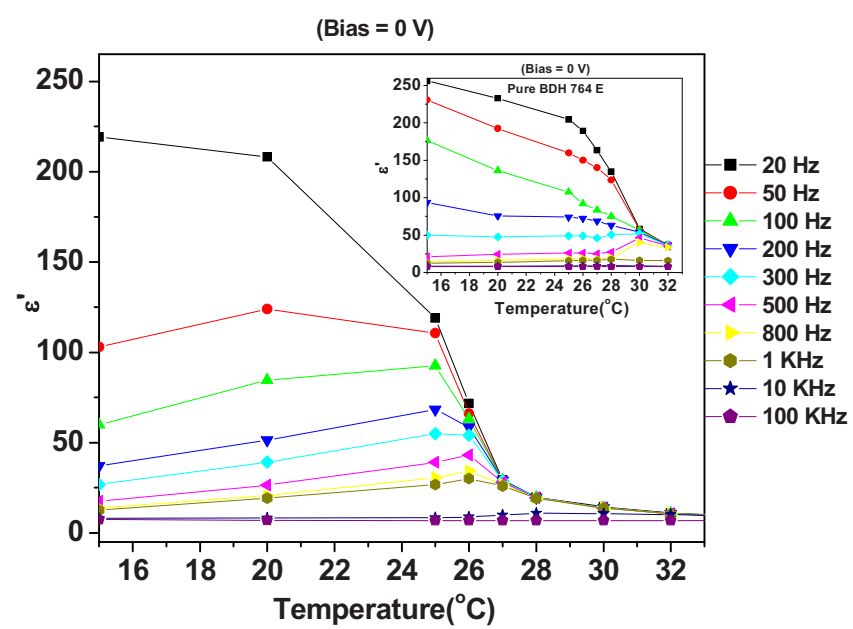

FIG. 2. (Color online) Dielectric permittivity $\left(\varepsilon^{\prime}\right)$ as a function of temperature for different frequency values under no bias for GO doped ELC (planar aligned under dc electric field) and in inset, pure ELC sample cells.

stant in both $\mathrm{SmC}^{*}$ and $\mathrm{SmA}^{*}$ phase of pure ELC at same frequency, $1 \mathrm{kHz}$, as shown in inset of Fig. 2 at $0 \mathrm{~V}$ bias. In deep $\operatorname{SmC}^{*}\left(15{ }^{\circ} \mathrm{C}\right)$ phase, the $\varepsilon^{\prime}$ value at frequency more than $20 \mathrm{~Hz}$ is lower and increases with temperature near $\mathrm{T}_{\mathrm{c}}$, but in case of pure ELC, it either decreases or remains constant near $T_{c}$. These results confirm the reduction in $T_{c}$ of GO doped ELC sample, i.e., the ordering in ELC has been perturbed by addition of $\mathrm{GO}$ nanomaterial as it can be seen from the behavior of low value of $\varepsilon^{\prime}$ near $\mathrm{T}_{\mathrm{c}}$. It shows the drop of $\varepsilon^{\prime}$ at $27^{\circ} \mathrm{C}$ in doped sample while this is at $29-30{ }^{\circ} \mathrm{C}$ in case of pure, inset of Fig. 2. The value of $\varepsilon^{\prime}$ at higher frequencies $10 \mathrm{k}-100 \mathrm{kHz}$ remains very low in both the cases.

Figure 3 shows the behavior of $\tan \delta$ versus frequency of GO doped ELC sample with temperature. However, the exact relaxation frequency can be calculated by using the relation $\nu_{\mathrm{R}, \varepsilon^{\prime \prime}}=\nu_{\mathrm{R}, \tan \delta} \sqrt{\varepsilon_{\infty} / \varepsilon_{o}},{ }^{34}$ where $\nu_{\mathrm{R}, \varepsilon^{\prime \prime}}$ and $\nu_{\mathrm{R}, \tan \delta}$ are the relaxation frequencies calculated from the values of $\varepsilon^{\prime \prime}$ and $\tan \delta$, respectively. The peaks in $\varepsilon^{\prime \prime}$ calculated with above formula shifts toward lower frequency due to high value of $\varepsilon^{\prime}$ at lower frequencies. The low frequency relaxation processes $\left(\nu_{\mathrm{R}, \varepsilon^{\prime \prime}}\right)$ peaks can be clearly seen in $\tan \delta$ versus frequency curves which is not observable in $\varepsilon^{\prime \prime}$ versus fre-

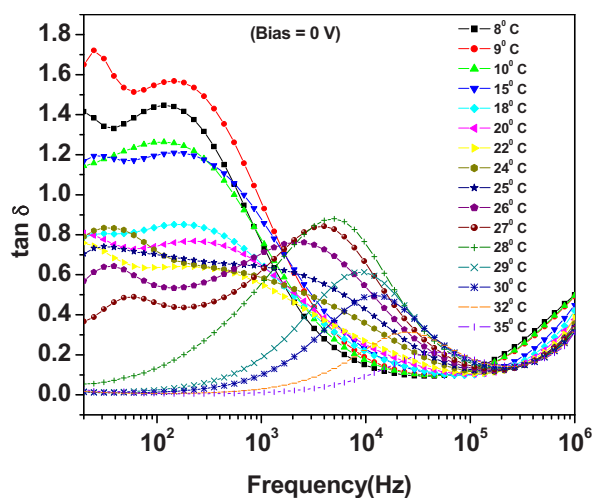

FIG. 3. (Color online) Frequency dependence of $\tan \delta$ (loss factor) measured in the temperature range $8-35^{\circ} \mathrm{C}$ in the planar aligned (under $\mathrm{dc}$ electric field) sample cell of GO doped ELC.

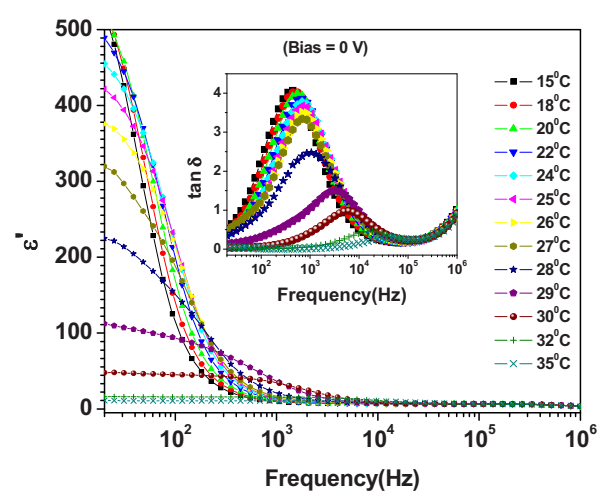

FIG. 4. (Color online) Dielectric permittivity $\left(\varepsilon^{\prime}\right)$ and $\tan \delta$ (in inset) as a function of frequency in the temperature range $15-35{ }^{\circ} \mathrm{C}$ under no bias for heptane (nondipolar) doped ELC sample cell.

quency (data not shown here). These two relaxation peaks have been observed in the GO added ELC sample (Fig. 3). The frequency separation of both peaks increases with temperature and the low frequency peak vanishes at $T_{c}$. After $T_{c}$, only the regular soft mode process remains there and shows the usual behavior. Such low frequency relaxation peak has also been observed recently in water added ELC samples ${ }^{35}$ and for nematic LC. ${ }^{36}$

In order to confirm the origin of the low frequency peak in $\tan \delta$ versus frequency, a nondipolar organic material (heptane) has been added in the same ELC material and in same aspect ratio of composition, as shown in Fig. 4. Inset of Fig. 4 shows no visualization of slow relaxation process. The relaxation process is almost similar and even transition temperature also looks to be same as in pure ELC sample. This observation shows that the slow process in GO doped ELC is due to dipolar contribution of dopant along with the main dipolar component of ELC.

The relaxation frequency $\left(\nu_{\mathrm{R}}\right)$, calculated by $\nu_{\mathrm{R}, \varepsilon^{\prime \prime}}$ $=\nu_{\mathrm{R}, \tan \delta} \sqrt{\varepsilon_{\infty} / \varepsilon_{o}}$, is plotted with respect to temperature for GO doped ELC and pure ELC in Fig. 5, in order to observe the behavior near $\mathrm{T}_{\mathrm{c}}$ in both the cases. The transition temperature of pure ELC is $28^{\circ} \mathrm{C}$, but it has been reduced about $1{ }^{\circ} \mathrm{C}$ in case of doped. After $\mathrm{T}_{\mathrm{c}}$ in $\mathrm{SmA}^{*}$ phase, the relaxation process in GO doped ELC sample at $0 \mathrm{~V}$ bias shows the appearance of soft mode process in two ways. First, the process from 25 to $28{ }^{\circ} \mathrm{C}$ is not showing the common be-

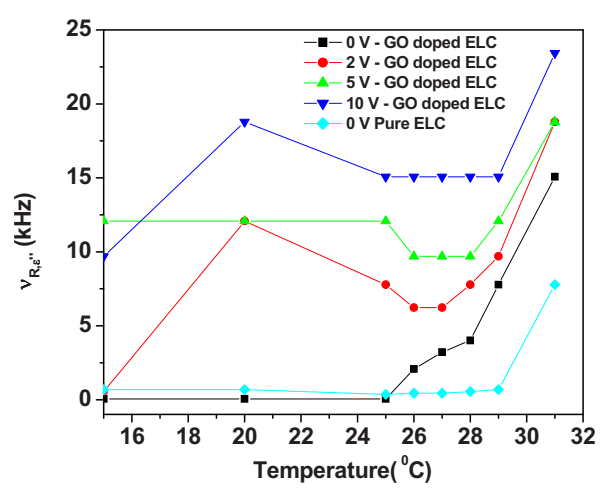

FIG. 5. (Color online) Behavior of relaxation frequency with bias 0, 2, 5, and $10 \mathrm{~V}$ for GO doped ELC and at $0 \mathrm{~V}$ for pure ELC at different temperatures. 

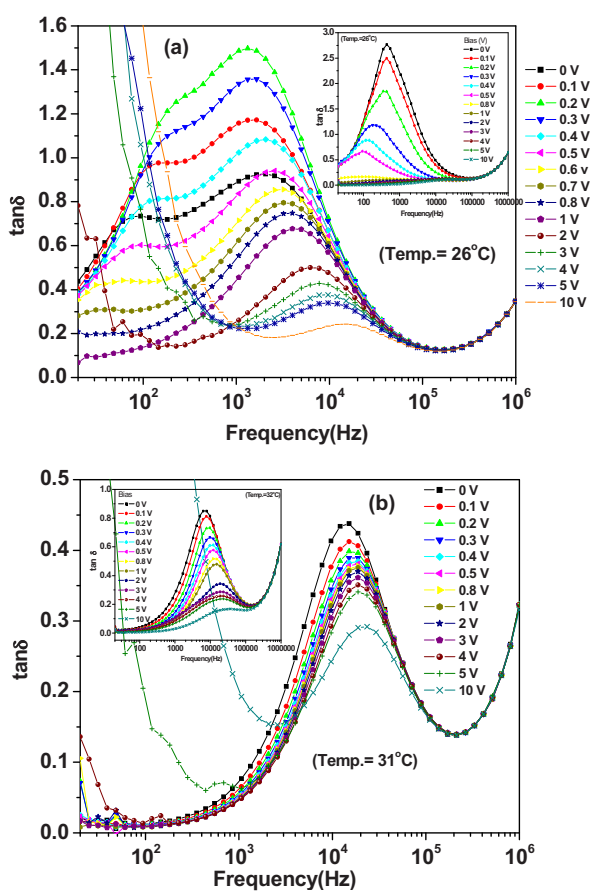

FIG. 6. (Color online) (a) Behavior of dielectric loss factor ( $\tan \delta$ ) of GO doped ELC and in inset, pure ELC material with frequency at different values of applied voltages at $26{ }^{\circ} \mathrm{C}\left(\mathrm{SmC}^{*}\right.$ phase). (b) Behavior of dielectric loss factor $(\tan \delta)$ of GO doped ELC and in inset, pure ELC material with frequency at different values of applied voltages at $31^{\circ} \mathrm{C}$ (SmA phase).

havior. But in second, the linear slope at higher temperatures from $28{ }^{\circ} \mathrm{C}$ shows the behavior similar to pure ELC. Such observation is also visible in Fig. 2 also at $1 \mathrm{kHz}$ frequency where the $\varepsilon^{\prime}$ value shows unusual behavior in the vicinity of $\mathrm{T}_{\mathrm{c}}$. This behavior of relaxation process could reflect the effect of GO coupling with ITO substrate and ELC material.

For further investigation, the bias dependent relaxation process in vertical to planar converted sample cell has been studied in $\mathrm{SmC}^{*}$ and $\mathrm{SmA}^{*}$ phases in both doped and pure materials. Figure 6(a) shows the behavior of $\tan \delta$ versus frequency curves with bias. The slow process frequency peak shows reduction in characteristic process whereas the general Goldstone mode process peak shows the increment with bias. The low frequency peak disappears completely at $0.8 \mathrm{~V}$ bias, which shows the complete suppression with bias and only Goldstone mode exists there. The inset of Fig. 6(a) shows the relaxation process peak in pure ELC sample which exhibits no slow relaxation process but a regular Goldstone mode is observable. Also in Fig. 6(b), again no low relaxation frequency peak is visible and only a soft mode process is observable in SmA* phase of both doped and pure BDH 764E [inset of Fig. 6(b)] which is the normal behavior in ELC materials. The appearance of low frequency process in $\mathrm{SmC}^{*}$ only suggests that the low frequency process is associated with $\mathrm{SmC}^{*}$ process, i.e., Goldstone mode.

Again in Fig. 6(a), the amplitude of $\tan \delta$ peak increases initially with bias up to $0.2 \mathrm{~V}$ and then decreases gradually, whereas in case of pure ELC, inset of Fig. 6(a), there is no such increment but a continuous reduction with bias. Since, the increment in the amplitude shows no change in shape of curve but a small frequency shifting of relaxation peaks. This behavior of enhancement in amplitude and shifting in relax-
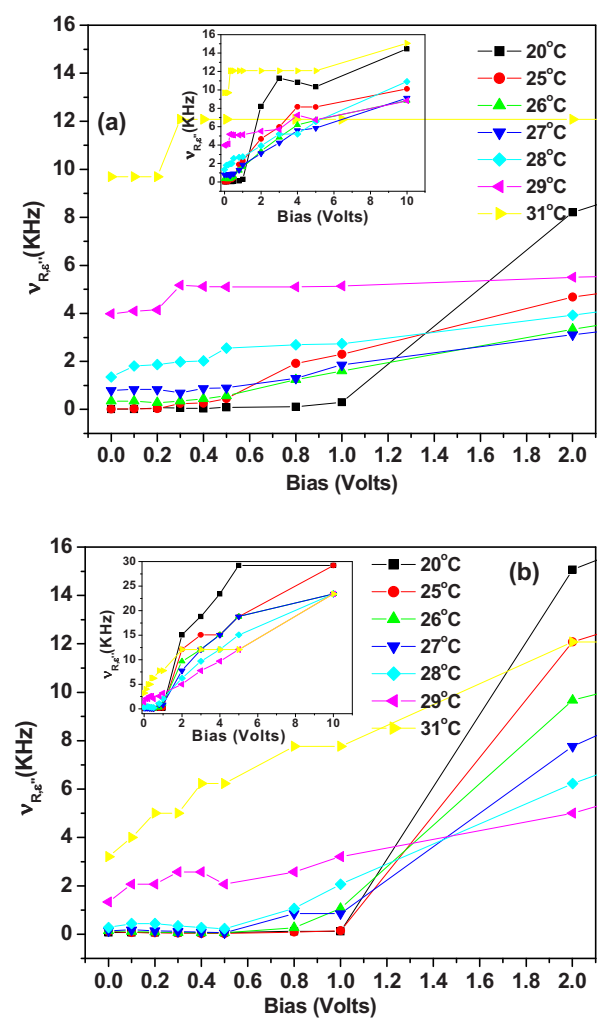

FIG. 7. (Color online) (a) Behavior of relaxation frequency with temperature for GO doped ELC for bias range $0-2 \mathrm{~V}$ and in inset, the same behavior for bias range $0-10 \mathrm{~V}$. (b) Behavior of relaxation frequency with temperature for pure ELC for bias range $0-2 \mathrm{~V}$ and in inset, the same behavior for bias range $0-10 \mathrm{~V}$.

ation peaks is due to the obvious contribution of GO in Goldstone mode process, i.e., the enhanced dipolar process contribution up to threshold bias and then, after a threshold value, it starts decreasing, i.e., the Goldstone mode process has been suppressed which is the normal behavior in ELCs. It is worth to mention here that above $100 \mathrm{kHz}$ the increment in $\tan \delta$ is due to the finite resistance of the ITO layer as seen in Fig. 6, which can be minimized by using gold coated electrodes.

Figure 7 shows the behavior of relaxation process with bias and at various temperatures in GO doped and pure ELC sample. Figure 7(a) shows the values as a function of bias up to $2 \mathrm{~V}$ at different temperatures while inset shows the data in the range of $0-10 \mathrm{~V}$ bias of GO doped ELC at various temperatures. In Fig. 7(b), the studies have been shown at same parameters of temperature in pure ELC sample and show the relaxation frequencies at $0-10 \mathrm{~V}$ bias range. In GO doped ELC sample, Fig. 7(a), the $\nu_{\mathrm{R}, \varepsilon^{\prime \prime}}$ increases with temperature up to $\mathrm{T}_{\mathrm{c}}\left(28^{\circ} \mathrm{C}\right)$ in $\mathrm{SmC}^{*}$ phase and after that it behaves as usual in $\mathrm{SmA}^{*}$ phase at $0-1 \mathrm{~V}$ bias. In pure ELC, Fig. 7(b), it remains unchanged in $\mathrm{SmC}^{*}$ phase as usual and increases continuously with temperature in $\mathrm{SmC}^{*}$ and $\mathrm{SmA}^{*}$ phase above $1 \mathrm{~V}$ bias. Consequently, the increasing behavior of $\nu_{\mathrm{R}}$ with temperature from $0-0.8 \mathrm{~V}$ bias shows the induced dipolar contribution of GO because the additional peak in $\tan \delta$ versus frequency curve also disappears up to $0.8 \mathrm{~V}$ bias in GO doped sample in $\mathrm{SmC}^{*}$ phase, as can be seen in Fig. 6(a).

The temperature independency of relaxation process shows the general behavior of Goldstone mode in SmC* 

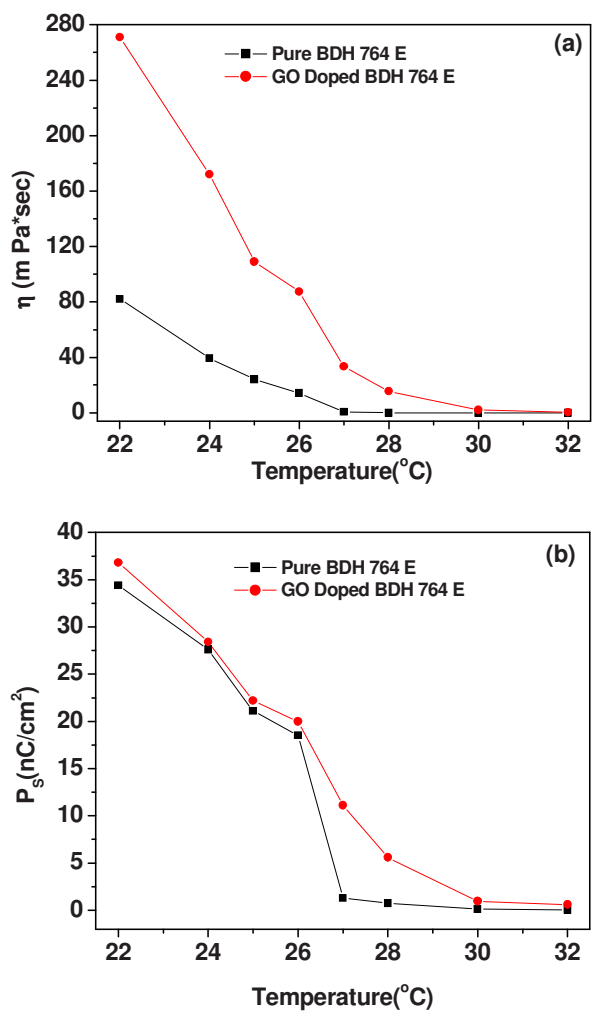

FIG. 8. (Color online) Behavior of (a) rotational viscosity $(\eta)$ and (b) induced polarization $\left(\mathrm{P}_{\mathrm{S}}\right)$ with temperature for both graphene oxide $(\mathrm{GO})$ doped and pure ELC materials.

phase. After a threshold of $0.8 \mathrm{~V}$ bias, the regular process remain almost independent of bias in GO doped sample while it becomes bias dependent after a threshold bias in both $\mathrm{SmC}^{*}$ and $\mathrm{SmA}^{*}$ phase of pure sample, as can be seen in both Figs. 7(a) and 7(b). The threshold bias increment of $\nu_{\mathrm{R}}$ variation decreases with temperature in GO doped sample, inset Fig. 7(a). But, it remains constant in $\mathrm{SmC}^{*}$ phase in pure BDH 764E (ELC) at $0 \mathrm{~V}$ bias.

Effect of GO addition on parameters such as induced polarization $\left(\mathrm{P}_{\mathrm{s}}\right)$ and rotational viscosity $(\eta)$ has been shown in Fig. 8. Figure 8(a) shows increment in $\eta$ value in GO doped sample and drops to minimum at $30{ }^{\circ} \mathrm{C}$ while in pure it drops to minimum at $27^{\circ} \mathrm{C}$. Its respective $\mathrm{P}_{\mathrm{s}}$ value versus temperature curve in Fig. 8(b) shows negligible increment in $\mathrm{P}_{\mathrm{s}}$ value in $\mathrm{SmC}^{*}$ phase. But minimization of $\mathrm{P}_{\mathrm{s}}$ at $30{ }^{\circ} \mathrm{C}$ shows the transition before $30{ }^{\circ} \mathrm{C}$ as compared to $\nu_{\mathrm{R}, \varepsilon^{\prime \prime}}$ versus temperature curve and matches with $\eta$ value in Fig. 8(a). In case of pure sample this is a general behavior of ELC materials. This could happen because the ELC material is highly electroclinic in nature near $\mathrm{T}_{\mathrm{c}}$. This can also reflect the effect of anchoring on the surface substrate without any alignment layer. The enhanced $\eta$ shows that the switching process in the sample, converted into planar alignment by the application of strong bias of $30 \mathrm{~V}$, may not be a complete conversion. This conversion might not be completely planar due to the vertical anchoring of ELC molecules with ITO substrate through GO nanomaterial. The possible interaction of oxide-oxide (graphene and indium tin oxide) and the affected molecular ordering (can be expected from reduced $\mathrm{T}_{\mathrm{c}}$ ) may result vertical anchoring.
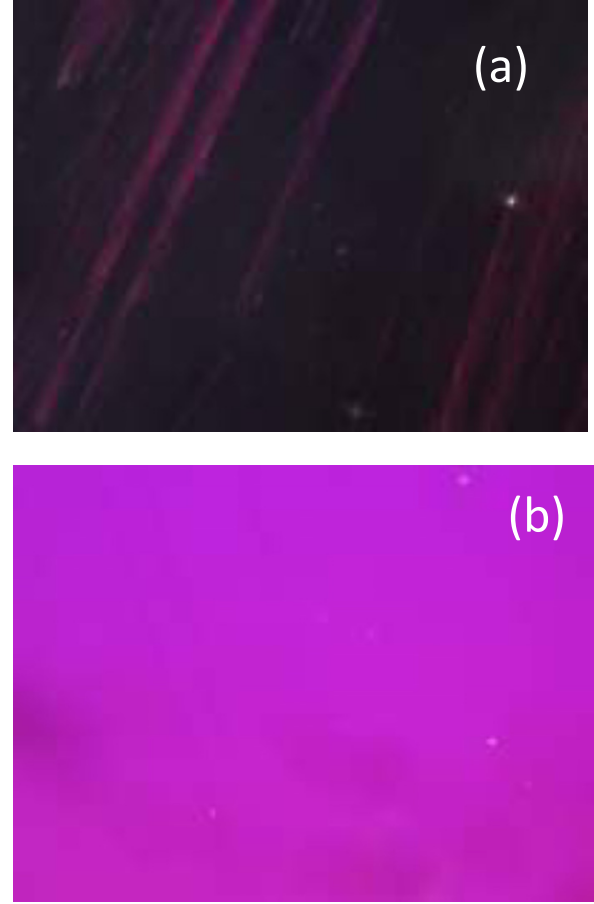

FIG. 9. (Color online) Optical micrographs (a) dark state and (b) bright state, of a planar aligned (nylon treated) sample cell of GO doped ELC material.

Furthermore, another measurement for comparison of substrate attachment with ELC has been carried out on the ITO substrate coated with nylon $6 / 6$ and rubbed with velvet cloth for proper planar alignment of ELC molecules. It is to examine whether the vertical alignment is due to ITO surface anchoring of molecules or any bulk induced effect. Figure 9 shows the relevant textures of planar alignment of GO doped ELC material filled in the treated sample cell, i.e., the attachment of ELC material is mediated by nylon 6/6 layer. The dark and bright state has been obtained in virgin sample cell, as shown in Figs. 9(a) and 9(b). Its $\varepsilon^{\prime}$ versus temperature curve for surface treated sample is shown in Fig. 10. At 1 $\mathrm{kHz}$ frequency near $\mathrm{T}_{\mathrm{c}}$ the $\varepsilon^{\prime}$ increases gradually and then decreases as in Fig. 2 (inset) for untreated sample. This exhibits the effect of GO in bulk and not on the surface. The major differences have been observed in an untreated sample where there is a direct exposure of ITO to GO-ELC sample.

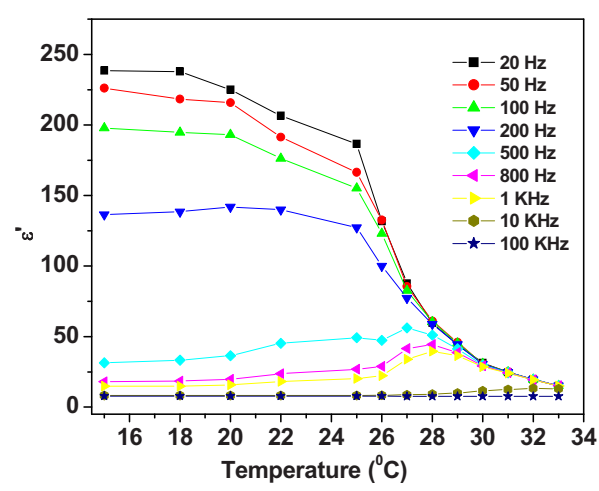

FIG. 10. (Color online) Dielectric permittivity $\left(\varepsilon^{\prime}\right)$ as a function of temperature for different frequency values under no bias for planer aligned (nylon treated) GO doped ELC sample cell. 
This means that the vertical alignment in untreated sample substrates is only due to the interaction of GO and ITO components. GO works as an order-affecting agent, which affects the viscosity of ELC and makes it easier to be aligned along the GO attached to the ITO surface in vertical direction. Hence, GO interacts with ITO layer and supports the ELC molecules to align in the $\mathrm{GO}$ directed direction.

\section{CONCLUSIONS}

It can be concluded that a perfect vertical alignment has been achieved in the sample cells without any surface treatment because of the coupling between ELC molecules and ITO film mediated by GO nanomaterial. Our results illustrate an additional low frequency relaxation peak along with Goldstone mode in $\mathrm{SmC}^{*}$ phase due to the presence of GO and the low frequency peak vanishes near transition temperature. The achievement of perfect vertical alignment for an ELC material has substantial prospective and the technique can be adopted for various dynamic studies of ELC materials.

\section{ACKNOWLEDGMENTS}

The authors sincerely thank Director, Professor R. C. Budhani, of National Physical Laboratory, for continuous encouragement and interest in this work. Authors (A.M. and A.C.) are thankful to CSIR, New Delhi for financial assistance.

${ }^{1}$ R. B. Meyer, L. Liebert, L. Strzelecki, and P. Keller, J. Phys. (Paris) Lett. 36, 69 (1975).

${ }^{2}$ T. Carlsson, B. Zeks, C. Filipic, and A. Levstik, Phys. Rev. A 42, 877 (1990).

${ }^{3}$ F. Gouda, G. Andersson, M. Matuszczyk, K. Skarp, and S. T. Lagerwall, J. Appl. Phys. 67, 180 (1990).

${ }^{4}$ J. Pavel, M. Glogarova, and S. S. Bawa, Ferroelectrics 76, 221 (1987).

${ }^{5}$ A. M. Biradar, S. Wrobel, and W. Haase, Phys. Rev. A 39, 2693 (1989).

${ }^{6}$ S. Garoff and R. B. Meyer, Phys. Rev. A 19, 338 (1979).

${ }^{7}$ S. Garoff and R. B. Meyer, Phys. Rev. Lett. 38, 848 (1977).

${ }^{8}$ F. Giesselmann, P. Zugenmaier, I. Dierking, S. T. Lagerwall, B. Stebler, M. Kaspar, V. Hamplova, and M. Glogarova, Phys. Rev. E 60, 598 (1999).

${ }^{9}$ J. P. F. Lagerwall, F. Giesselmann, and M. D. Radcliffe, Phys. Rev. E 66 , 031703 (2002).

${ }^{10}$ A. Tang, D. Konovalov, J. Naciri, B. R. Ratna, and S. Sprunt, Phys. Rev.
E 65, 010703 (2001)

${ }^{11}$ M. S. Spector, P. A. Heiney, J. Naciri, B. T. Weslowski, D. B. Holt, and R. Shashidhar, Phys. Rev. E 61, 1579 (2000).

${ }^{12}$ G. Andersson, I. Dahl, W. Kuczynski, S. T. Lagerwall, K. Skarp, and B. Stebler, Ferroelectrics 84, 285 (1988).

${ }^{13}$ A. Levstik, T. Carlsson, C. Filipic, I. Levstik, and B. Zeks, Phys. Rev. A 35, 3527 (1987).

${ }^{14}$ A. M. Biradar, D. Kilian, S. Wrobel, and W. Haase, Liq. Cryst. 27, 225 (2000).

${ }^{15}$ S. A. Róźański and J. Thoen, Liq. Cryst. 32, 331 (2005).

${ }^{16}$ Y. Shiraishi, N. Toshima, K. Maeda, H. Yushikawa, J. Xu, and S. Kobayashi, Appl. Phys. Lett. 81, 2845 (2002).

${ }^{17}$ J. Prakash, A. Choudhary, A. Kumar, D. S. Mehta, and A. M. Biradar, Appl. Phys. Lett. 93, 112904 (2008).

${ }^{18}$ J. Prakash, A. Choudhary, D. S. Mehta, and A. M. Biradar, Phys. Rev. E 80, 012701 (2009).

${ }^{19}$ W. Lee, C. Y. Wang, and Y. C. Shih, Appl. Phys. Lett. 85, 513 (2004).

${ }^{20}$ A. Kumar, J. Prakash, D. S. Mehta, A. M. Biradar, and W. Haase, Appl. Phys. Lett. 95, 023117 (2009).

${ }^{21}$ W. S. Hummers, Jr. and R. E. Offeman, J. Am. Chem. Soc. 80, 1339 (1958).

${ }^{22}$ H. A. Becerril, J. Mao, Z. Liu, R. M. Stoltenberg, Z. Bao, and Y. Chen, ACS Nano, 2, 463 (2008).

${ }^{23}$ G. Eda, G. Fanchini, and M. Chhowalla, Nat. Nanotechnol. 3, 270 (2008).

${ }^{24}$ X. Wang, L. Zhi, N. Tsao, Z. Tomovic, J. Li, and K. Mullen, Angew. Chem., Int. Ed. 47, 2990 (2008).

${ }^{25}$ S. Watcharotone, D. A. Dikin, S. Stankovich, R. Piner, I. Jung, G. H. B. Dommett, G. Evmenenko, S. E. Wu, S. F. Chen, C. P. Liu, S. T. Nguyen, and R. S. Ruoff, Nano Lett. 7, 1888 (2007).

${ }^{26}$ S. Stankovich, D. A. Dikin, G. H. B. Dommett, K. M. Kohlhaas, E. J. Zimney, E. A. Stach, R. D. Piner, S. T. Nguyen, and R. S. Ruoff, Nature (London) 442, 282 (2006).

${ }^{27}$ P. Blake, P. D. Brimicombe, R. R. Nair, T. J. Booth, D. Jiang, F. Schedin, L. A. Ponomarenko, S. V. Morozov, H. F. Gleeson, E. W. Hill, A. K. Geim, and K. S. Novoselov, Nano Lett. 8, 1704 (2008).

${ }^{28}$ A. Safavi and M. Tohidi, J. Phys. Chem. C 114, 6132 (2010).

${ }^{29}$ F. Kim, L. J. Cote, and J. Huang, Adv. Mater. (Weinheim, Ger.) 22, 1954 (2010).

${ }^{30}$ V. K. Singh, M. K. Patra, M. Manoth, G. S. Gowd, S. R. Vadera, and N. Kumar, New Carbon Mater. 24, 147 (2009).

${ }^{31}$ S. Kaur, A. K. Thakur, R. Chauhan, S. S. Bawa, and A. M. Biradar, J. Appl. Phys. 96, 2547 (2004).

${ }^{32}$ F. Kremer and A. Schoenhals, Broadband Dielectric Spectroscopy (Springer, Berlin, 2002)

${ }^{33}$ A. Kumar, J. Prakash, P. Goel, T. Khan, S. K. Dhawan, P. Silotia, and A. M. Biradar, EPL 88, 26003 (2009).

${ }^{34}$ A. Chelkowski, Dielectric Physics (Elsevier, New York, 1980).

${ }^{35}$ G. Singh, A. Choudhary, G. V. Prakash, and A. M. Biradar, Phys. Rev. E 81, 051707 (2010).

${ }^{36}$ G. Meier and A. Saupe, Mol. Cryst. Liq. Cryst. 1, 515 (1966). 